\title{
Deletion of GSK-3 $\beta$ in mice leads to hypertrophic cardiomyopathy secondary to cardiomyoblast hyperproliferation
}

\author{
Risto Kerkela, ${ }^{1}$ Lisa Kockeritz, ${ }^{2}$ Katrina MacAulay, ${ }^{2}$ Jibin Zhou, ${ }^{1}$ Bradley W. Doble,, ${ }^{2}$ \\ Cara Beahm, ${ }^{1}$ Sarah Greytak, ${ }^{3}$ Kathleen Woulfe, ${ }^{1}$ Chinmay M. Trivedi, ${ }^{4}$ \\ James R. Woodgett, ${ }^{2}$ Jonathan A. Epstein, ${ }^{4}$ Thomas Force, ${ }^{1}$ and Gordon S. Huggins ${ }^{3}$ \\ ${ }^{1}$ Center for Translational Medicine, Thomas Jefferson University, Philadelphia, Pennsylvania, USA. 2Samuel Lunenfeld Research Institute, \\ Mount Sinai Hospital, Toronto, Ontario, Canada. ${ }^{3}$ Molecular Cardiology Research Institute, Tufts Medical Center, Boston, Massachusetts, USA. \\ 4Penn Cardiovascular Institute, University of Pennsylvania, Philadelphia, Pennsylvania, USA.
}

\begin{abstract}
Based on extensive preclinical data, glycogen synthase kinase-3 (GSK-3) has been proposed to be a viable drug target for a wide variety of disease states, ranging from diabetes to bipolar disorder. Since these new drugs, which will be more powerful GSK-3 inhibitors than lithium, may potentially be given to women of childbearing potential, and since it has controversially been suggested that lithium therapy might be linked to congenital cardiac defects, we asked whether GSK-3 family members are required for normal heart development in mice. We report that terminal cardiomyocyte differentiation was substantially blunted in $\mathrm{Gsk} 3 \mathrm{~b}^{-/-}$embryoid bodies. While GSK-3 $\alpha$-deficient mice were born without a cardiac phenotype, no live-born $\mathrm{Gsk} 3 b^{-/-}$pups were recovered. The Gsk $3 b^{-/-}$embryos had a double outlet $\mathrm{RV}$, ventricular septal defects, and hypertrophic myopathy, with near obliteration of the ventricular cavities. The hypertrophic myopathy was caused by cardiomyocyte hyperproliferation without hypertrophy and was associated with increased expression and nuclear localization of three regulators of proliferation - GATA4, cyclin D1, and c-Myc. These studies, which we believe are the first in mammals to examine the role of GSK-3 $\alpha$ and GSK-3 $\beta$ in the heart using loss-of-function approaches, implicate GSK-3 $\beta$ as a central regulator of embryonic cardiomyocyte proliferation and differentiation, as well as of outflow tract development. Although controversy over the teratogenic effects of lithium remains, our studies suggest that caution should be exercised in the use of newer, more potent drugs targeting GSK-3 in women of childbearing age.
\end{abstract}

\section{Introduction}

The factors regulating proliferation of cardiomyocytes during development have been the focus of many investigations in the past decade. These studies have identified several growth factors, acting in a paracrine fashion, that regulate cardiac growth and chamber morphogenesis. Specifically, endothelium-derived neuregulin-1 (Nrg1), acting via its receptor and coreceptor, the tyrosine kinases Erb-B4 and Erb-B2, is critical for chamber morphogenesis and trabeculation of the ventricular walls (1-3). Other studies have identified IGF-1 (4), retinoic acid, FGF9, -16, and -20 (5), and BMP10 (6) as regulators of cardiomyocyte proliferation during mid-gestation. These factors likely regulate cardiomyocyte proliferation through transcription factors, including $\mathrm{N}$-myc, GATA4, T-box genes, and, possibly, nuclear factor of activated $\mathrm{T}$ cells (NF-AT) family members (in particular, NF-ATc3 and NF-ATc4) (7-11). Signaling pathways connecting growth factors and these downstream mediators of proliferation are poorly understood. Not surprisingly, the PI3K pathway, which is the primary signaling arm of several growth factor receptors, including IGF-1, has been found to play a role in cardiomyocyte proliferation ex vivo and in vitro $(4,12,13)$. However, it remains unclear

Nonstandard abbreviations used: DORV, double outlet RV; EB, embryoid body; GSK, glycogen synthase kinase; NF-AT, nuclear factor of activated T cells; TNFR1, TNF- $\alpha$ receptor-1; VSD, ventricular septal defect.

Conflict of interest: The authors have declared that no conflict of interest exists. Citation for this article: J. Clin. Invest. 118:3609-3618 (2008). doi:10.1172/JCI36245 whether this is relevant in vivo, which specific components of this complex pathway might regulate growth, and how they accomplish this, particularly in the developing heart.

The glycogen synthase kinase isoforms GSK-3 $\alpha$ and GSK-3 $\beta$ have nearly identical kinase domains but differ in their $\mathrm{N}$ - and C-terminal sequences $(14,15)$. These kinases are key regulators of the canonical Wnt pathway through phosphorylation-mediated targeting of $\beta$-catenin for proteasomal degradation (16-19). The Wnt/ $\beta$-catenin pathway plays a central role in endocardial cushion and atrioventricular valve development via regulating the process of endothelial-mesenchymal transition $(\mathrm{EMT})(16,19)$. Indeed, proper regulation of canonical Wnt pathway components, including dishevelled-2, is required for outflow tract development (20, 21). However, isoform-specific roles for GSK-3 $\alpha$ and $-3 \beta$ in Wnt/ $\beta$-catenin-regulated heart development are unlikely, since both kinases are functionally redundant in regulating $\beta$-catenin stability and 3 of 4 GSK-3 alleles must be disrupted before an effect on $\beta$-catenin signaling can be found (22).

GSK-3 kinases have also been reported to negatively regulate several transcription factors and basic cell cycle regulators implicated in heart development, such as D-type cyclins and Myc family members $(23,24)$. However, the studies suggesting a role for GSK-3 in regulating these factors are largely based on overexpression approaches in cultured cell lines, and, recently, some have challenged the putative role played by GSK-3 (25-27).

We believed that determining the role played by GSK-3 in cardiac development was an important issue in and of itself, but the 
Table 1

Expression of differentiation markers in EBs

\begin{tabular}{|c|c|c|c|}
\hline Marker & WT, $n=3$ & Gsk3a-/-, $n=3$ & Gsk3b-/-, $n=3$ \\
\hline Nanog & $2.19 \pm 0.16$ & $1.82 \pm 0.19$ & $1.57 \pm 0.11$ \\
\hline Brachyury & $2.53 \pm 0.28$ & $3.26 \pm 0.66$ & $0.82 \pm 0.18^{A}$ \\
\hline GATA4 & $0.88 \pm 0.27$ & $0.89 \pm 0.13$ & $0.89 \pm 0.03$ \\
\hline$N k \times 2.5$ & $2.13 \pm 0.74$ & $3.13 \pm 0.46$ & $1.08 \pm 0.20$ \\
\hline$\beta-M H C$ & $4.69 \pm 0.46$ & $2.39 \pm 0.56^{B}$ & $0.93 \pm 0.07^{A, B}$ \\
\hline$\alpha-M H C$ & $5.69 \pm 0.95$ & $3.42 \pm 0.45^{B}$ & $0.87 \pm 0.17^{\mathrm{A}, \mathrm{B}}$ \\
\hline SERCA2 & $2.43 \pm 0.15$ & $1.96 \pm 0.24$ & $1.03 \pm 0.05^{A, B}$ \\
\hline$B N P$ & $6.18 \pm 0.87$ & $4.25 \pm 0.23$ & $1.18 \pm 0.12^{\mathrm{A}, \mathrm{B}}$ \\
\hline
\end{tabular}

Values are arbitrary units (mean \pm SEM). ${ }^{A} P<0.05$ versus Gsk3a ${ }^{-1-}$; ${ }^{\mathrm{B} P}<0.05$ versus WT. BNP, B-type natriuretic peptide; $\beta$-MHC, $\beta$-myosin heavy chain; SERCA2, sarcoplasmic reticulum $\mathrm{Ca2}^{+}$-ATPase.

question may take on greater importance given the proposed use of small molecule inhibitors of GSK-3 in women of childbearing age who suffer from bipolar disorder. It is estimated that nearly $4 \%$ of the US population suffers from bipolar disorder (28), and inhibition of GSK-3 is believed to play a key role in the therapeutic response to lithium treatment (29), which has been the mainstay of therapy for 50 years. Based on the preponderance of evidence (reviewed in ref. 29), GSK-3 has been identified as a viable target for treatment of bipolar disorder. Since there is a suggestive link between lithium therapy and congenital cardiac malformations (30), the active development of newer GSK-3 inhibitors that are significantly more potent than lithium increases the importance of defining the role of GSK-3 in cardiac development.

To determine the role of GSK-3 kinases in general, and specific isoforms in particular, in mammalian heart development, we studied ES cells and mice deleted for either Gsk3a or Gsk3b. While the GSK-3 $\alpha$-deficient embryos were born at the expected frequency and had no cardiac developmental defects, no live-born GSK- $3 \beta$-deficient mice were recovered. Some GSK-3 $\beta$-deficient embryos died in mid-gestation due to severe liver degeneration, as previously described (31). However, penetrance of this phenotype was not uniform, and the majority of animals survived to late gestational periods (32). In these embryos, many of which appeared grossly normal upon inspection, we demonstrate a profound effect of deletion of GSK-3 $\beta$ on proliferative growth in the developing heart and a key role for the kinase in normal development of the outflow tract. This is the first report to our knowledge of cardiac development in mice deficient of either GSK- $3 \alpha$ or GSK-3 $\beta$ and should heighten awareness of the potential risks involved in inhibiting these kinases in the developing embryo.

\section{Results}

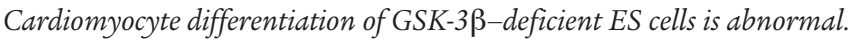
To determine the role of the GSK-3 isoforms in cardiomyocyte differentiation, we induced embryoid body (EB) formation using the hanging-drop method with the previously characterized WT,

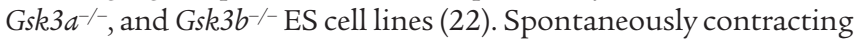
WT and Gsk3a-l- EBs were found after 7 days. However, beating Gsk $3 b^{-/-}$EBs were not found until day 8. Compared with WT (82 beating per $93 \mathrm{EBs}, 88 \%)$, there were significantly fewer beating Gsk3a-l- EBs ( 66 beating per $90 \mathrm{EBs}, 73 \% ; P=0.01$ versus WT) and many fewer contracting Gsk3b-/- EBs (43 beating per $96 \mathrm{EBs}, 44 \%$; $P<0.0001$ versus WT and versus Gsk3a- $a^{--}$.
To determine whether the delayed onset of $G s k 3 b^{-/-}$EB contraction correlated with differences in genes upregulated during cardiomyocyte differentiation, quantitative PCR was performed. Similar levels of Nanog were found in the 3 cell lines after differentiation; however, brachyury, a marker of mesoderm development, was reduced in $\mathrm{Gsk} 3 b^{-1-}$ compared with WT and Gsk3a-/- EBs (Table 1). Expression of the transcription factors GATA4 and Nkx2.5, which mark the earliest stages of cardiomyocyte differentiation, was similar. In contrast, expression of the contractile proteins $\alpha$-myosin heavy chain $(\alpha-\mathrm{MHC})$ and $\beta$-MHC, later markers of cardiomyocyte differentiation, was significantly reduced in the Gsk3-/- EBs, with the greatest effect found in the Gsk $3 b^{-1-} \mathrm{EBs}$ (Table 1). Sarcoplasmic reticulum $\mathrm{Ca}^{2+}$-ATPase (SERCA2), an important regulator of cardiomyocyte contraction whose expression is strongest after birth, marks late cardiomyocyte differentiation. Expression of SERCA2, as well as the B-type natriuretic peptide (BNP), was selectively reduced in $\mathrm{Gsk} 3 b^{-/-}$EBs. These data, taken together, suggest that GSK-3 $\beta$ is necessary for late cardiomyocyte differentiation.

GSK-3ß-deficient embryos have congenital heart defects. To determine the role of GSK-3 proteins in cardiac development and to examine isoform-specific effects, we produced Gsk3a-/- mice by gene targeting and compared their cardiac development with that of the GSK-3 $\beta$-deficient mouse. Gsk3a-/ mice are fertile and are born at expected Mendelian ratios with no cardiac developmental abnormalities (Supplemental Figure 1; supplemental material available online with this article; doi:10.1172/JCI36245DS1). By comparison, no live-born $\mathrm{Gs} 3 b^{-/-}$animals were recovered.

Previously, Gsk3b-/ embryos were found to be susceptible to liver failure in utero. More recently, however, Liu et al. did not observe a significant increase in mid-gestational embryonic death in Gsk $3 b^{-/-}$ embryos (32), which would be inconsistent with liver failure. To explore more deeply the timing of $G s k 3 b^{-/}$embryo demise, and the prevalence of liver failure, we genotyped 287 embryos ranging from E12.5 to E19.5 derived from heterozygote matings. While fewer Gsk $3 b^{-1-}$ mice were found than expected, the number was not significantly different from that predicted for mid-and late-gestational periods (Table 2). Markedly pale Gsk3b-/ embryos, which are characteristic of liver failure, were infrequently recovered during mid- and late-gestational periods (32). Thus, GSK-3 $\beta$, and not GSK- $3 \alpha$, is necessary for survival after birth.

Since liver failure did not explain the lack of viability of Gsk $3 b^{-1-}$ pups, we tested for congenital defects in heart development that can be responsible for perinatal death. At E13.5, Gsk $3 b^{+/+}$embryos showed the expected pattern of cardiac outflow tract septation (dividing the truncus arteriosus into the aorta and pulmonary artery), positioning of the aorta over the left ventricle, connection of the pulmonary artery to the right ventricle, and near or complete closure of the interventricular septum. By comparison, in E13.5-E17.5 Gsk $3 b^{-/-}$embryos, the aorta and pulmonary artery consistently arose from the RV (double outlet RV [DORV]) and a ventricular

\section{Table 2}

Survival of mutant embryos

\begin{tabular}{llccc} 
Time point & Gsk3b+/ & Gsk3b+/- & Gsk3b-/- & $\boldsymbol{\chi}^{\mathbf{2}} \boldsymbol{P}$ value \\
E12.5-E15.5 & 51 & 99 & 40 & NS \\
E16.5-E19.5 & 23 & 55 & 19 & NS \\
\hline
\end{tabular}

Values indicate total number of embryos genotyped at each gestational age. 

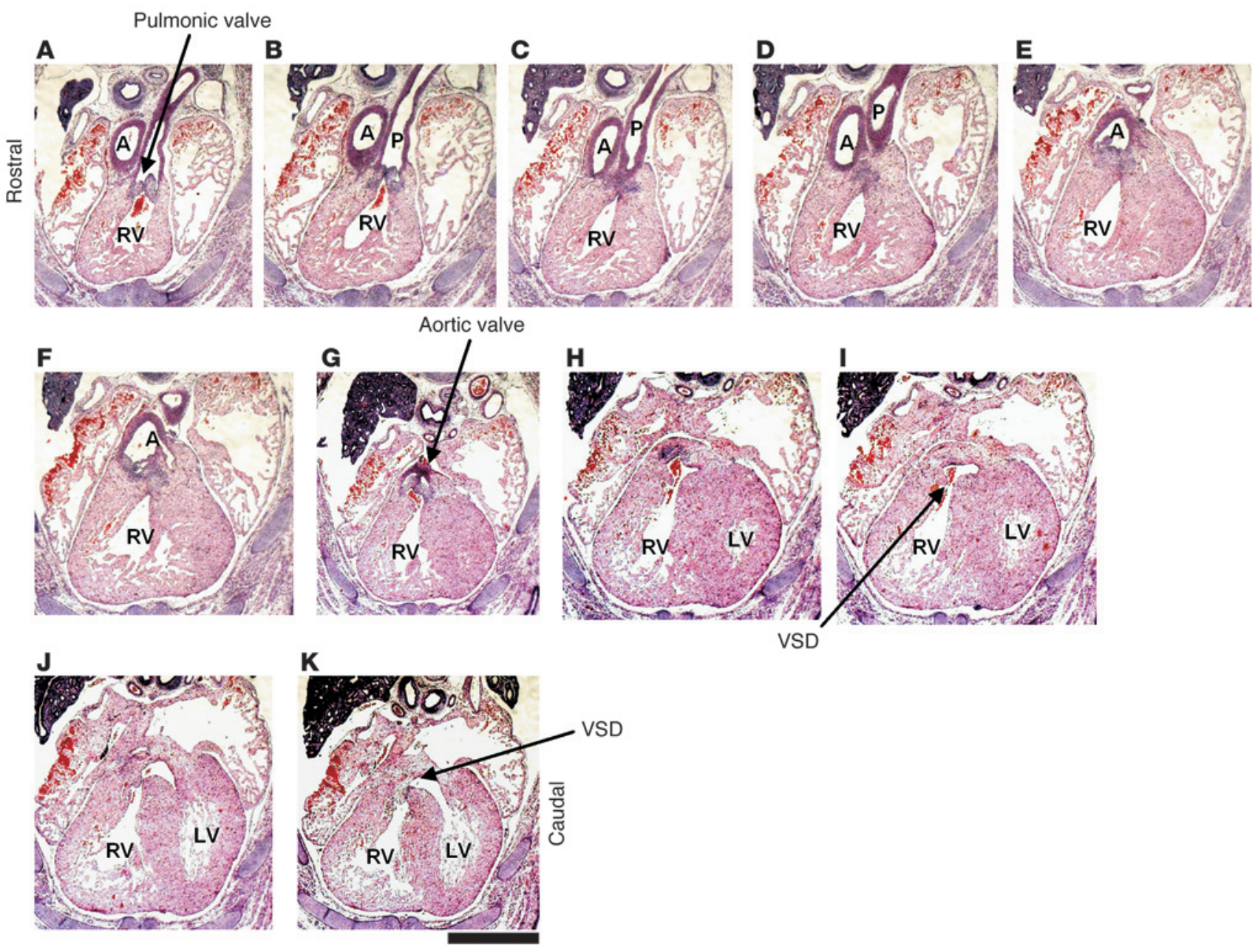

VSD

\section{Figure 1}

Histological analysis of heart development in GSK-3 $\beta$-deficient mice. Sequential rostral-to-caudal sections from an E15.5 Gsk3b-/- embryo from the pulmonary valve (top left) to the ventricles (bottom right) (original magnification, $\times 2.0$; scale bar: $0.5 \mathrm{~cm}$ ). The pulmonary valve and the pulmonary artery $(\mathrm{P})$ are positioned over the RV $(\mathbf{A}-\mathbf{C})$. Below the pulmonary valve, the aorta $(\mathrm{A})$ descends toward the aortic valve $(\mathbf{D}-\mathbf{F})$; the aortic valve is positioned over the RV (G). Caudal to the semilunar valves, the RV and the LV are connected by a VSD (H-K). The location of the VSD is in the membranous portion of the interventricular septum.

septal defect (VSD) was apparent (Figure 1). DORV and VSD can be the product of abnormal cardiac cushion development; however, with the exception of the VSD, the endocardial cushion-derived valves and septae appeared normal in Gsk3b-/- animals (Figure 2, $A$ and $B$ ). Defects of cardiac neural crest cell migration or function that produce DORV and VSD commonly also produce abnormal remodeling of the cardiac outflow tract and aortic arch arteries (e.g., interrupted aortic arch). However, no abnormalities of great vessel development were observed in $G s k 3 b^{-/-}$embryos $(n=13)$. Furthermore, expression of the cardiac neural crest markers plexin A2 and semaphorin 3C was not different in WT and Gsk3b-/- embryos (Supplemental Figure 2). These results demonstrate that GSK-3 $\beta$, and not GSK-3 $\alpha$, is required for normal cardiac outflow tract development and suggest that the DORV is not due to abnormalities of the endocardial cushions or neural crest.

Gsk3b-null embryos have markedly thickened ventricular walls. The late gestation stage of embryo development is characterized by remodeling of the ventricular chambers. These changes in heart shape, which we consider an example of physiologic "remodeling," include greater organization of the myocardial layers and gross enlargement of the ventricular cavities to accommodate a larger stroke volume.

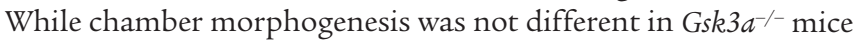
compared with WT at 2 days after birth (Supplemental Figure 1), by E15.5 (Figure 1) and at E17.5, Gsk36 $b^{-/}$embryos displayed markedly thickened LV and RV walls (Figure 2, A-C). Compared with those of WT littermates, ventricular cavities of $\mathrm{Gsk} 3 \mathrm{~b}^{-/-}$embryos were smaller and nearly filled with cells (Figure 2, A and B). We also noted pulmonary blood congestion in Gsk $3 b^{-/}$embryos (Figure $2 \mathrm{D}$ ), which is consistent with embryonic heart failure, and this likely contributed to perinatal death.

Previously, GSK-3 $\beta$ deficiency has been shown to sensitize cells to the effects of TNF (31). To exclude a role for enhanced TNF sensitivity in the observed myocardial changes, we crossed the Gsk3b-/- line with mice lacking TNF- $\alpha$ receptor-1 (TNFR1) (33). As was the case with $G s k 3 b^{-/-}$single-gene knockout, no live-born

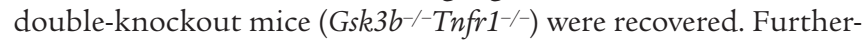


A

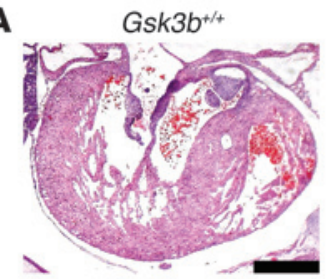

B

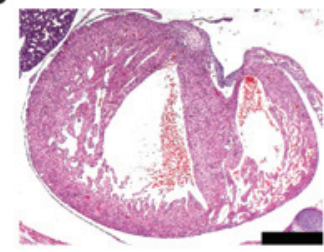

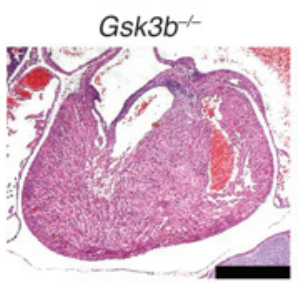

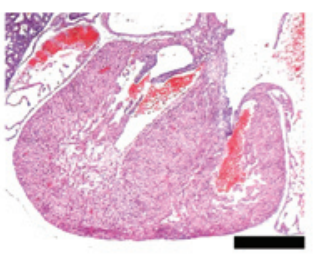

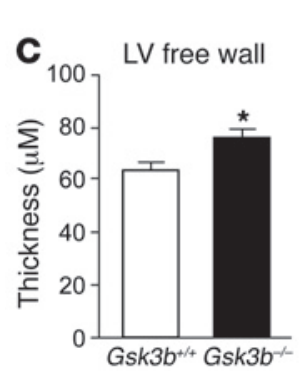
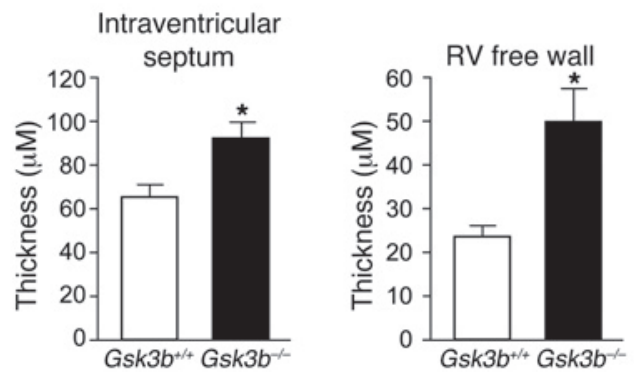

D

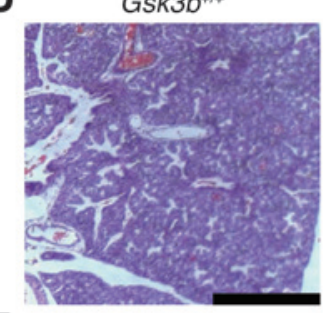

E

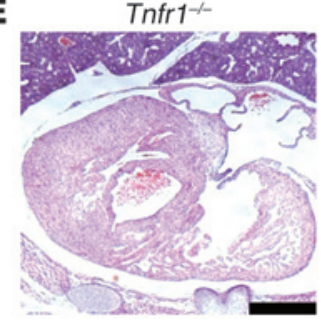

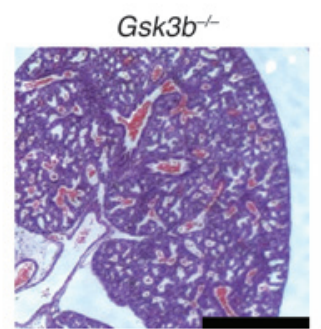

Gsk3b-1-Tnfr 1-1-

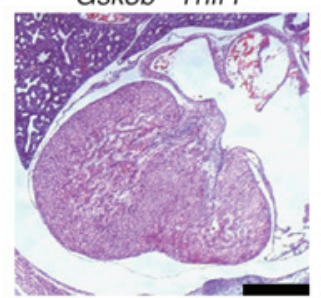

Figure 2

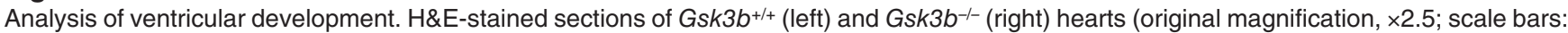
$0.1 \mathrm{~mm}$ ) including the mitral valve leaflet $(\mathbf{A})$ and the tricuspid valve leaflet $(\mathbf{B})$ (original magnification, $\times 2.5$; scale bars: $0.5 \mathrm{~mm}$ ). The atrioventricular valve leaflets of Gsk3b/- embryos showed cellularity, chordal attachment, and dimension comparable to those of control embryos. By comparison, the LV wall thickness was greatly increased in the Gsk3b-/- embryo hearts. (C) Quantification of thickness of the LV free wall,

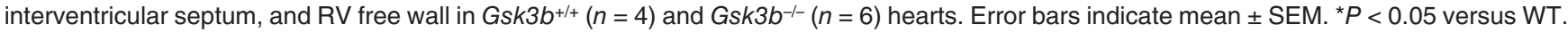
(D) The pulmonary vasculature and parenchyma showed congestion of blood in Gsk3 $b^{-/-}$(right) compared with Gsk3b//+ (left) embryos (original

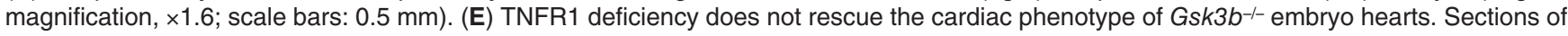
Tnfr1 $^{-/-}$(left) and Gsk3b ${ }^{-/-}$Tnfr1 $^{-/-}$(right) embryos, including the tricuspid valve leaflet (original magnification, $\times 1.6$; scale bars: $0.5 \mathrm{~mm}$ ).

more, E17.5 double-knockout animals demonstrated increased myocardial thickness compared with $\mathrm{Tnfr} 1^{-/-}$control embryos (Figure 2E). These studies suggest an isoform-specific role for GSK-3 $\beta$ in cardiac hypertrophy and outflow tract development that is independent of TNF signaling.

Cardiomyocyte byperproliferation accounts for the increased wall thickness in GSK-3ß-deficient hearts. The overwhelming majority of the myocardial cells in $\mathrm{Gs} k 3 b^{-/-}$embryo hearts stained positive for GATA4 but negative for the endothelial cell-specific marker isolectin B4 (data not shown), consistent with hyperproliferation and/or hypertrophy, specifically of cardiomyocytes, accounting for the increase in wall thickness. Based on studies employing overexpression of GSK-3 $\beta$, it appears that the kinase negatively regulates both physiologic and pathologic postnatal cardiomyocyte hypertrophy (34-37). To determine whether the loss of GSK-3 $\beta$ caused hypertrophy, we measured cardiomyocyte size in GSK-3 $\beta$-deficient embryos, employing staining with FITC-conjugated wheat germ agglutinin (Supplemental Figure $3 \mathrm{~A})$. There was a statistically significant, but very modest, increase in cardiomyocyte cross-sectional area in the Gsk3b-/- embryos at E15.5 (Supplemental Figure 3B) that was not apparent at E17.5 (data not shown). Furthermore, while the GSK-3 proteins are critical regulators of glycogen storage, there was no difference in glycogen content in Gsk3b $b^{--}$and WT embryos (Supplemental Figure 3C). Thus, cellular hypertrophy and glycogen accumulation could not account for the enhanced wall thickness of the Gsk3b-/- embryos at E17.5.
Since cardiomyocyte hypertrophy did not explain the observed wall thickening, we explored whether the phenotype could be secondary to either reduced apoptosis or increased proliferation. We saw no significant differences between WT and Gsk $3 b^{-/-}$mice in rates of cardiomyocyte apoptosis as determined by TUNEL assay (Supplemental Figure 4). In contrast, we observed a marked increase in levels of phosphorylated histone H3 (an M phase-specific marker) at both E13.5 and E15.5 in the myocardium of Gsk3b-embryos, consistent with enhanced proliferation (Figure 3, A and B). To determine whether the increased proliferation seen was specifically due to cardiomyocyte proliferation, we costained embryo sections for both phosphorylated histone H3 and GATA4. The vast majority of cells (>90\%) staining positive for phospho-histone $\mathrm{H} 3$ were also positive for GATA4, suggesting that they were proliferating cardiomyocytes (Figure 3C). Cardiac ECs can also express GATA4 (38). However, ECs, identified by isolectin B4 staining, accounted for only $6 \%$ and $8 \%$ of phospho-histone $\mathrm{H} 3$-positive

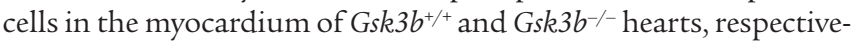
ly. Thus, the increased proliferation in the Gsk $3 b^{-/-}$hearts is due to hyperproliferation of cardiomyocytes and this, in turn, appears to account for the thickened myocardium at late gestation.

GSK-3 $\beta$ deficiency alters expression and/or nuclear partitioning of cardiac cell cycle regulators. To explore the mechanism by which GSK-3 $\beta$ deficiency may increase cardiomyocyte proliferation, we examined GSK-3 targets implicated in heart development for altered expres- 
A
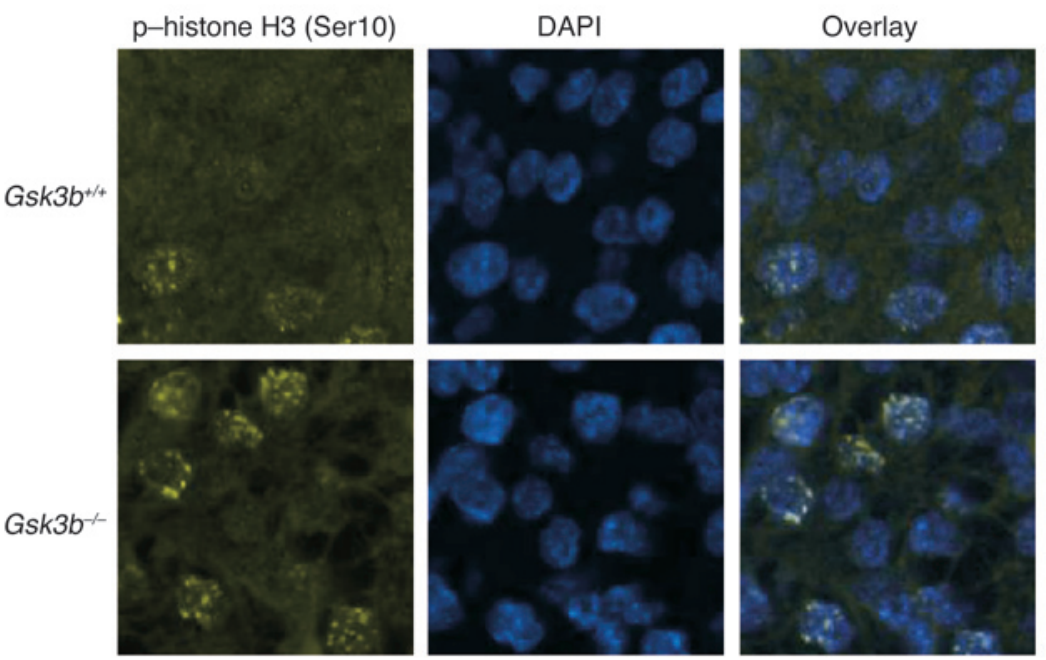

B

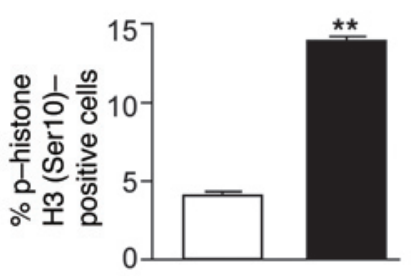

E13.5

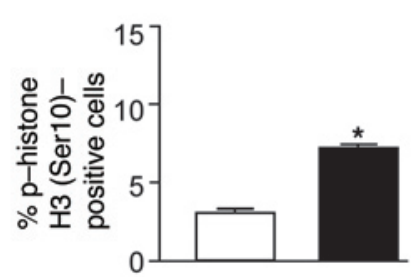

E15.5

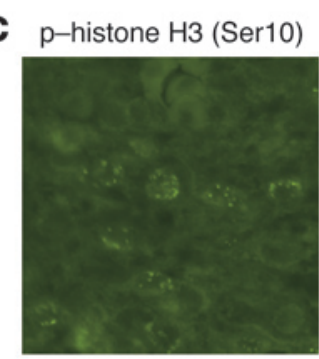

GATA4

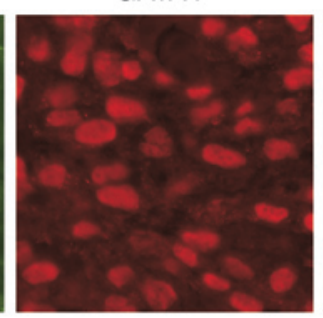

Overlay

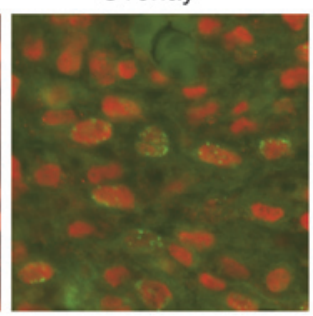

$\square$ Gsk3b/t+

Gsk3b ${ }^{-1-}$

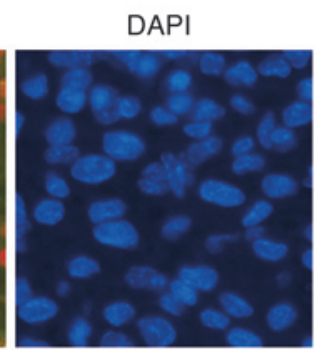

\section{Figure 3}

Increased cardiomyocyte proliferation in GSK-3 $\beta$-deficient hearts. (A) Immunofluorescence staining for nuclear phospho-histone $\mathrm{H} 3$ (Ser10) (left panels) in GSK-3 $\beta-$ deficient (bottom) compared with WT (top) animals. Merging of the histone and the DAPI (middle) stains confirmed nuclear localization of the phospho-histone $\mathrm{H} 3$ (Ser10) staining (right panels). Original magnification, $\times 40$. (B) Composite mean \pm SEM percentage of phospho-histone $\mathrm{H} 3$ (Ser10)-staining cells at E13.5 and E15.5 in the myocardium of WT (white bars) and GSK-3 $\beta$-deficient (back bars) animals. ${ }^{*} P<0.05$, ${ }^{* *} P<0.001$ versus WT. (C) Coimmunostaining of heart sections with phospho-histone $\mathrm{H} 3$ and GATA4, a cardiomyocyte marker, demonstrating colocalization in the overlay. Thus, the phospho-histone H3 (Ser10)-positive cells in the myocardium are overwhelmingly GATA4 positive, consistent with cardiomyocytes. Also shown is nuclear staining with DAPI. Original magnification, $\times 40$. sion and/or nuclear/cytoplasmic partitioning in the Gsk3- $3 b^{-/-}$ embryos. Because GSK-3 negatively regulates $\beta$-catenin, we asked whether enhanced $\beta$-catenin signaling in cardiomyocytes might account in part for the growth phenotype of the Gsk3 $\mathrm{b}^{-/-}$embryos. However, $\beta$-catenin was overwhelmingly associated with the cell membrane in ventricular myocytes at E13.5 (Supplemental Figure $5 \mathrm{~A}$ ) and E15.5 (data not shown) and in the nucleus of valve-forming regions, irrespective of genotype (Supplemental Figure 5B).

Contradictory reports have appeared addressing whether GSK-3 $\beta$ regulates stability of various cyclins $(23,25-27)$. Therefore, we analyzed sections of multiple $G s k 3 b^{-/-}$and $G s k 3 b^{+/+}$embryonic hearts with immunohistochemistry, focusing on the amount of cyclin D1 staining present in the nucleus. Indeed, increased cyclin D1 was present in the nucleus of Gsk $3 b^{-/-}$compared with $G s k 3 b^{+/+}$embryos. To determine the significance of this difference, we asked whether an independent observer could predict the genotype based upon the level of expression of nuclear cyclin D1. The genotype of 10 of 11 $G s k 3 b^{-1-}$ and $G s k 3 b^{+/+}$heart sections was correctly predicted on the basis of increased nuclear cyclin D1 staining in the knockout $(P<0.01$; Figure 4A). Thus, our findings support the concept that GSK-3 $\beta$ does regulate cyclin D1, at least in the developing heart, and provide one potential mechanism contributing to the hyperproliferative phenotype. We also examined cyclin E, which has been reported to

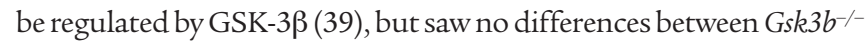
and WT littermates (data not shown). Myc family members also drive cardiomyocyte proliferation, and c-Myc and N-Myc levels are regulated by GSK-3 $\beta$ (24). The genotype of 9 of 11 heart sections was correctly predicted based on an increase in nuclear c-Myc $(P<0.05$; Figure 4B) but not N-myc (data not shown) in cardiomyo-

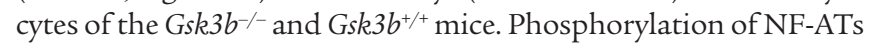
by GSK-3 $\beta$ leads to cytosolic localization (40); however, we could detect no obvious enhancement of nuclear-localized NF-ATc3 or NF-ATc4 in Gsk3b-/- cardiomyocytes (data not shown).

Finally, we examined GATA4 nuclear partitioning, as a study employing overexpression of a dominant-negative mutant of GSK-3 $\beta$ in vitro suggested that GSK-3 $\beta$ regulates nuclear GATA4 levels (41). We found a marked increase in nuclear-localized GATA4 in the absence of GSK-3 $\beta$ (Figure 5) and correctly identified the genotype of 16 of 16 heart sections based on the amount of nuclear GATA4 present $(P<0.01)$. These data suggest that partitioning and/or expression of GATA 4 is regulated by GSK-3 $\beta$ in vivo.

Inbibition of GSK-3 and GATA4 overexpression drives cardiomyocyte proliferation. To explore the role of GSK-3 and GATA4 in driving cardiomyocyte proliferation, we examined the effect of pharmacologic inhibition of GSK-3 and adenovirus-mediated gene transfer of GATA4 on proliferation of cultured neonatal rat car- 
A

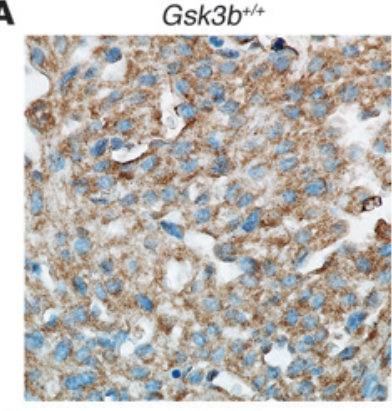

B

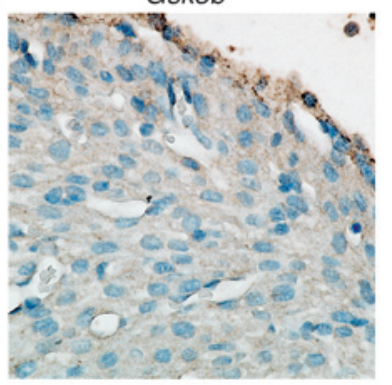

Gsk3b-

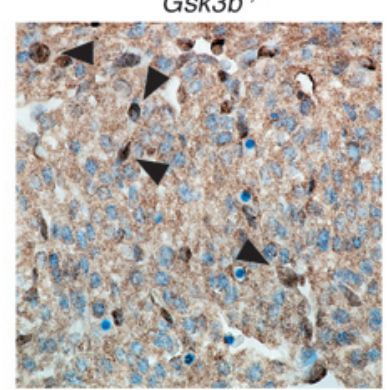

Gsk3b ${ }^{--}$

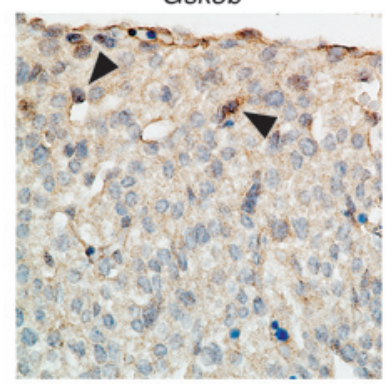

diomyocytes, which retain the ability to proliferate for 2-3 days after isolation. The GSK-3 inhibitors lithium chloride and 6-bromoindirubin-3'-oxime (BIO) significantly increased proliferation as determined by phospho-histone H3 staining (Figure 6A). Similarly, even modest overexpression of GATA4 was sufficient to drive cardiomyocyte proliferation (Figure 6B). These studies confirm a cell-autonomous effect of GSK-3 inhibition and GATA4 expression on cardiomyocyte proliferation. Consistent with GSK-3 $\beta$ having several targets regulating cell cycle progression in addition to GATA4 (e.g., cyclin D1 and c-Myc), GSK-3 inhibition induced more pronounced proliferation than GATA4 gene transfer.

\section{Discussion}

Herein we report that ablation of GSK-3 $\beta$ led to impaired cardiomyocyte differentiation in ES cells and to pronounced hyperplasia of cardiomyocytes during embryonic development, culminating in a ventricle filled with myocytes. In striking contrast, defects of cardiomyocyte differentiation of Gsk3a-/-ES cells were less severe, and cardiac development in the Gsk3a-/- mouse was normal. These data clearly demonstrate distinct developmental roles of the two GSK-3 isoforms. Furthermore, our studies identify disordered regulation of GATA4, cyclin D1, and c-Myc, three factors known to play a role in cardiomyocyte proliferation during development, in the Gsk3b-/- heart and implicate these factors in the hyperproliferative phenotype.

We detected similar expression of GATA4 and Nkx2.5 in GSK-3-deficient EBs, which suggests that regulation of early cardiomyocyte differentiation is not specific to either GSK-3 isoform. This finding may be explained by the fact that $\beta$-catenin signaling, which promotes induction of the cardiomyocyte fate during the early stage of ES cell differentiation (42), is preserved in GSK-3 $\beta$ -

\section{Figure 4}

Altered transcription factor and cell cycle regulator expression and localization in GSK-3 $\beta$-deficient hearts. (A) Increased nuclear cyclin D1 in hearts of E15.5 GSK-3 $\beta$-deficient embryos. Sections were stained with anti-cyclin D1 antibody (brown) and then counterstained with hematoxylin to identify nuclei (blue). Note the multiple dark brown nuclei (arrowheads) and the overall increase in cyclin D1 stain and corresponding decrease in intensity of the hematoxylin stain in the GSK-3 $\beta-$ deficient hearts. Original magnification, $\times 40$. (B) $c-M y c$ expression is increased in GSK-3 $\beta$-deficient hearts. Sections from E15.5 embryos were stained with anti-c-Myc antibody (brown) and then counterstained with hematoxylin as described above. Note the enhanced brown staining of the nuclei (arrowheads) and the reduction in intensity of the hematoxylin stain, consistent with increases in nuclear c-Myc expression in the GSK-3 $\beta$-deficient heart. Original magnification, $\times 40$.

and GSK-3 $\alpha$-deficient EBs. Indeed, the lack of an increase in cytosolic or nuclear $\beta$-catenin in cardiomyocytes of Gsk3b-/ animals is consistent with the finding that Gsk3a can fully compensate for loss of Gsk3b in the regulation of $\beta$-catenin (22). By comparison, EB contraction and expression of late markers of cardiomyocyte differentiation were prominently reduced in GSK-3 $\beta$ deficient compared with GSK-3 $\alpha$-deficient and WT EBs. The pronounced inhibition of cardiomyocyte differentiation in the setting of GSK-3 $\beta$ deficiency suggests that GSK-3 $\beta$-selective regulation of transcription factors or cell cycle regulators is critical for the late stage of cardiomyocyte differentiation.

Differences in GSK-3 $\alpha$ and - $3 \beta$ signaling in cardiomyocyte differentiation are even more apparent in heart development. Although atrioventricular and semilunar valve development was normal in the Gsk $3 b^{-1-}$ mouse, outflow tract and septation defects were evident (DORV and VSD). The finding of DORV in GSK-3 $\beta$-deficient embryos may be interpreted as a nonspecific finding, as septation of the great vessels, creation of AV valves and outflow tracts, and closure of the VSD are regulated by cells of the endocardial cushions and neural crest. While abnormalities of neural crest could explain the DORV in GSK-3 $\beta$-deficient embryos (and only a tissue-specific knockout in neural crest cells could definitively address this), we
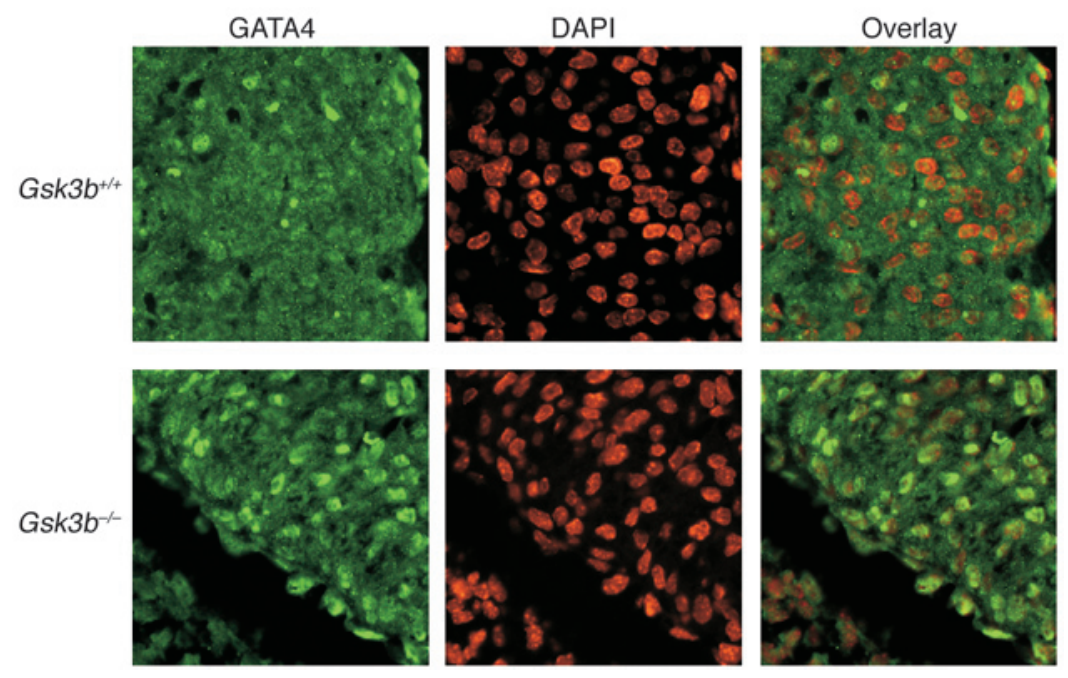

\section{Figure 5}

Increased nuclear GATA4 expression in GSK-3 $\beta$-deficient hearts. GATA4 staining (left) was located over nuclei in the hearts of E13.5 embryos stained by DAPI (center) in merged images (right). Original magnification, $\times 40$. 
A

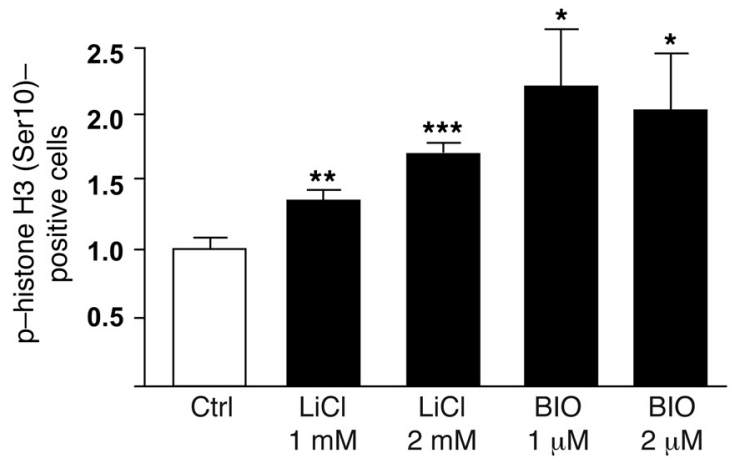

B

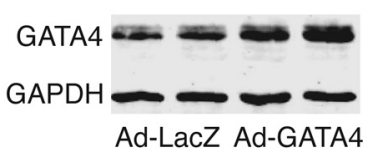

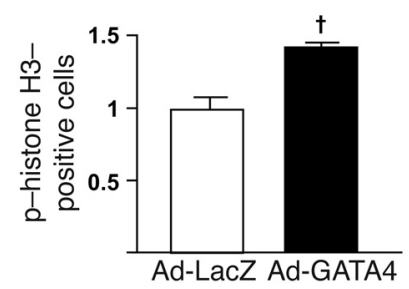

saw no evidence of improper septation of the great vessels, which is a common feature of neural crest defects. Furthermore, spatial migration of neural crest cells appeared normal. Enlargement of the endocardial cushions that impair normal rotation of the developing outflow tract can produce DORV. However, since there was no abnormality in valve development (which requires intact $\beta$-catenin signaling for the EMT), we consider a selective defect in the cushion cells to be unlikely. We considered that the marked distortion of LV geometry secondary to the cardiomyocyte hyperproliferation may have given rise to the DORV by impairing normal rotation of the outflow tract; however, the DORV was present before noticeable differences in ventricular wall thickness (data not shown).

Our finding that GSK-3 $\alpha$ is not required for mouse development is in contrast to observations in zebrafish, in which morpholino oligonucleotide-mediated knockdown of GSK-3 $\alpha$ produced severe axial developmental defects and death (43). Furthermore, while knockdown of either zebrafish GSK-3 isoform produced thin hearts and pericardial edema, we only found heart defects in $G s k 3 b^{-1-}$ embryos. Finally, while zebrafish GSK-3 $\alpha$ is required for cardiomyocyte survival, in striking contrast, we found that Gsk $3 b^{-/-}$ mouse embryos showed increased cardiomyocyte proliferation. We cannot reconcile the differences between the zebrafish and mouse loss-of-function studies; however, we speculate that differences in amino acid sequences flanking the serine/threonine kinase domain (44), altered substrate availability, or differences in the developmental expression pattern may account for unique roles for the GSK-3 isoforms in zebrafish compared with mammals. Despite these differences, morpholino oligonucleotide targeting of zebrafish GSK-3 $\beta$ produced abnormalities of heart tube jogging and outflow tract positioning (43) that may provide insight into the DORV observed in $\mathrm{Gsk}_{3} \mathrm{~b}^{-/-}$mice. The zebrafish outflow abnormalities correlated with abnormal lefty and BMP4 expression, suggesting an important role for GSK-3 $\beta$ in left-right patterning. Our data demonstrating DORV likewise suggest a requirement for GSK-3 $\beta$ in outflow tract development of the mammalian heart.

The striking enlargement of ventricular walls in late gestational periods, virtually filling the ventricular cavities, must be viewed in comparison to findings of studies by our group $(34,45)$ and oth-

\section{Figure 6}

GATA4 overexpression drives cardiomyocyte proliferation. (A) GSK-3 inhibition increases neonatal rat ventricular myocyte (NRVM) proliferation. Shown is the mean \pm SEM percentage of phospho-histone $\mathrm{H} 3$ (Ser10)-staining cells 20 hours following $\mathrm{LiCl}$ and 6-bromoindirubin3 '-oxime (BIO) treatment at the indicated concentrations. (B) GATA4 overexpression induces NRVM proliferation. Immunoblot of lysates from NRVMs infected with adenovirus encoding LacZ (left 2 lanes) or GATA4 (right 2 lanes). NRVMs were treated with 100 viral particles per cell (first and third lanes) or 200 particles per cell (second and fourth lanes). NRVMs plated onto glass coverslips were stained for phosphohistone H3 (Ser10) 48 hours after infection of cells with adenoviruses (200 viral particles per cell) encoding GATA4 (Ad-GATA4) or LacZ (Ad-LacZ). Shown is the mean \pm SEM percentage of phospho-histone H3 (Ser10)-staining cells following Ad-LacZ or Ad-GATA4 infection. ${ }^{\star} P<0.05,{ }^{* \star} P<0.01$, ${ }^{\star \star \star} P<0.001$ versus vehicle control (Ctrl); † $P<0.001$ versus Ad-LacZ.

ers $(35-37,46)$ that have employed overexpression approaches to suggest that GSK-3 $\beta$ is a potent negative regulator of cardiomyocyte growth. The growth that has been studied to date is postnatal growth, the vast majority of which is via hypertrophy rather than hyperplasia, since postnatal cardiomyocytes are generally unable to reenter the cell cycle except under extreme circumstances. Indeed, because cardiomyocyte cross-sectional areas in $\mathrm{Gsk} 3 \mathrm{~b}^{-/-}$and control E17.5 embryos were similar, the increased ventricular wall thickness is not the result of cardiomyocyte hypertrophy. Herein we identify a key role for GSK-3 $\beta$ in regulating hyperplastic growth of the developing heart. Because both the left and right ventricles were excessively cellular, relative to the chamber dimension, this finding is much more likely to be due to effects of deletion of GSK- $3 \beta$ than a response to an epigenetic influence (e.g., constrained outflow). Similarly, pharmacologic inhibition of GSK-3 also increased cardiomyocyte proliferation. Withdrawal from the cell cycle is required for the late and terminal stages of cardiomyocyte differentiation (47). Thus, active cycling of the Gsk3b-/- embryo cardiomyocytes is likely one important factor contributing to inhibition of their terminal differentiation.

The most striking consequences of GSK-3 $\beta$ deficiency that likely drive cardiomyocyte proliferation are increases in nuclear GATA4, cyclin D1, and c-Myc, all of which, based on our findings, appear to be targets of GSK-3 $\beta$ in vivo. GSK-3-mediated phosphorylation of cyclin D1 at Thr286 has been proposed to trigger nuclear export of cyclin D1 and priming of cyclin D1 for ubiquitination and degradation (23), though this is a subject of debate (25). Our data, from studies employing genetic loss of function of GSK- $3 \beta$ in vivo, support a role for GSK-3 $\beta$ as a regulator of cyclin D1 nuclear/cytoplasmic partitioning and degradation. Of note, the phenotype of the GSK-3 $\beta$ knockout is virtually the opposite of that of mice deleted for cyclins D1/D2/D3 or deleted for cyclin D partners Cdk2/Cdk4, which have marked thinning of the walls $(48,49)$. Interestingly, most tissues in the cyclin D1/D2/D3-deficient mouse had normal proliferation rates, while cardiomyocytes and hematopoietic stem cells appeared to be relatively unique in their dependence on D-type cyclins for driving proliferation (48).

Although GSK- $3 \beta$ deficiency affects several cell cycle regulators, its regulation of GATA-dependent signaling may be a critical contributor to cardiomyocyte proliferation. GATA4/GATA6 compound heterozygosity (50) and cardiomyocyte-specific knockout of GATA4 (11) produce heart developmental abnormalities characterized by reduced cardiomyocyte proliferation. Because the increased wall thickness in 
Gsk $3 b^{-/-}$embryos is related to hyperplasia, rather than hypertrophy, we propose that increased GATA4 nuclear accumulation in Gsk3b-1embryos can be expected to drive proliferation. Indeed, deletion of GSK-3 $\beta$ likely produces a GATA4 gain of function, in vivo, which drives proliferation. Our conclusion is supported by the finding that forced GATA4 expression (and GSK-3 inhibition) increases neonatal cardiomyocyte proliferation. Our results suggest that GSK-3 $\beta$-mediated phosphorylation and inhibition of GATA 4 and other regulators of cellular proliferation are necessary to restrict the number of cardiomyocytes during late gestational periods of heart development, which allows for completion of cardiomyocyte differentiation.

GSK-3 has been proposed as a viable target in numerous disease states, including ischemic injury of the heart and brain (51), inflammation (52), diabetes (53), Alzheimer disease (51), and bipolar disorders (29). While lithium is currently the only available approved drug that can inhibit GSK-3, numerous inhibitors with greater potency and specificity are in development (54). The issue of whether lithium, and by extension GSK-3 inhibition, causes congenital heart defects has been debated in the literature for more than 20 years because early retrospective analyses that reported high rates of congenital heart defects in the setting of lithium treatment were flawed (30). A more recent report confirmed a marginally increased risk for a cardiac defect after maternal use of lithium (55), and administration of lithium to animal models at very early gestational stages produces heart defects (56). However, it has been difficult to determine whether the heart defects observed in these lithium studies can be extrapolated to newer, more potent GSK-3 inhibitors for several reasons. First, lithium levels achieved in patients are only approximately $1 \mathrm{mM}$, and this causes (at physiological levels of $\mathrm{Mg}^{2+}$ ) only an approximately $25 \%$ inhibition of total GSK-3 activity (i.e., approximately equal to deletion of 1 allele of either GSK-3 $\alpha$ or GSK-3 $\beta$ ) (57). Second, lithium has several targets in addition to GSK-3, including Akt (which is activated by lithium and obviously has numerous targets in addition to GSK-3) and inositol monophosphatases (58). Indeed, depletion of intracellular inositol has been suggested to mediate the anti-manic effects of lithium (59). More recently, lithium has been shown to disrupt a complex consisting of Akt, GSK-3, protein phosphatase $2 \mathrm{a}$, and $\beta$-arrestin (60). Thus, lithium has effects well beyond simply inhibiting GSK-3, making it nearly impossible to ascribe specific effects of lithium to one specific target and completely impossible to ascribe effects to one specific isoform. We believe that our findings using genetically targeted mice bring clarity to the central issue: that inhibition of GSK-3 $\beta$ in the developing heart might be expected to lead to cardiac developmental abnormalities.

In summary, we have identified GSK-3 $\beta$ as a central regulator of cardiomyocyte proliferation and differentiation during embryonic heart development, mediated, at least in part, through its effects on 2 transcription factors and 1 cell cycle regulator. Deficits of ventricular remodeling of $\mathrm{Gs} k 3 b^{-/-}$animals are likely secondary to the requirement of GSK-3 $\beta$ to restrict myocardial cell number, but GSK- $3 \beta$ may also have a more direct role in maturation of the ventricle. The requirement for GSK-3 $\beta$ in heart development raises the consideration that mutations of GSK-3 $\beta$ may contribute to cases of DORV and VSD as well as LV hypertrophy in humans.

\section{Methods}

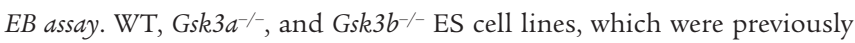
described (22), were maintained in Knockout DMEM supplemented with 15\% FBS (Hyclone), nonessential amino acids, GlutaMAX-I (Invitrogen), 2-mercaptoethanol, and 1,000 U/ml ESGRO (Chemicon). Differentiation medium for the production of embryoid bodies was embryonic stem cell maintenance medium with the serum content reduced to $5 \%$ and no ESGRO supplementation. EBs were propagated in hanging drops for 3 days and were then transferred to ultra-low binding 96-well dishes (Corning) and cultured with differentiation medium that was replenished every 2 days, as previously described (22). The EBs were examined for spontaneous beating daily. For each cell line, 3 pools of EBs were collected for expression analysis.

Quantitative real-time RT-PCR. cDNA was synthesized from $1 \mu \mathrm{g}$ total RNA using SuperScript III reverse transcriptase and oligo-dT primers (Invitrogen). For real-time PCR amplification, cDNA was diluted 1:100, and $1 \mu \mathrm{l}$ was used for each PCR amplification in triplicate with SYBR Green PCR Master Mix (Applied Biosystems) and $1 \mu \mathrm{M}$ transcript-specific primer (Supplemental Methods). Amplification and analysis were performed using an Applied Biosystems 7900 real-time PCR machine and SDS 2.3 software (Applied Biosystems). Products were sequenced to verify specificity of the primers. PCR efficiency was calculated from the slope of the respective standard. Fold changes in gene expression were determined using the Pfaffl method (61) and normalized to GAPDH after correcting for differences in amplification efficiency.

GSK-3-deficient mice. The generation of Gsk3b-/- mice has been previously described (31). Gsk3a-/- mice were generated by insertion of loxP sites flanking exon 2 of Gsk3a through homologous recombination with an engineered targeting construct. Germline deletion of exon 2 was accomplished by crossing the floxed mice with a PCAGG-Cre deleter strain (62) as described previously (22). By breeding Cre-deleted GSK-3 $\alpha$ heterozygous animals together, we generated Gsk3a-knockout, Gsk3a-heterozygous, and WT littermate control mice. In contrast to Gsk $3 b^{-/-}$embryos, which die late in gestation or immediately at birth, Gsk3a-/- mice were both viable and fertile. The genotype of embryos harvested following timed mating of heterozygote animals was determined by PCR as described previously $(22,31)$. TNFR1-deficient mice kindly provided by Tak Mak (33) were crossed with Gsk $3 b^{+/-}$mice to produce Gsk $3 b^{-/-}$Tnfr $1^{-/-}$double-knockout and $T n f r 1^{-/-}$control embryos. All animal experiments were approved by the Animal Care Committees of the Toronto Centre for Phenogenomics and the Ontario Cancer Institute under the auspices of the University of Toronto.

Cell size measurement. Cardiomyocyte cross-sectional area in E15.5 and E17.5 embryos was measured from 5 - $\mu$ m-thick heart sections that had been stained with FITC-conjugated wheat germ agglutinin as previously described (63), using OpenLab software-(Improvision). Only myocytes that were round (i.e., were cut in cross section) were included in the analysis. An observer blind to the genotypes of the embryos performed the measurements.

Histological analysis. Embryos were fixed for 18-24 hours in 4\% paraformaldehyde at $4{ }^{\circ} \mathrm{C}$, dehydrated in increasing concentrations of ethanol, and embedded in paraffin. Transverse and frontal sections were cut at $5 \mu \mathrm{m}$ through the thorax and stained with H\&E by standard methods. To determine the orientation of anatomic structures and analysis of embryonic heart development, serial sections through the heart and great vessel regions were imaged with an AxioCam MRc digital camera on a Zeiss Stemi 2000-c stereomicroscope. Each serial image was compiled to allow for analysis of heart chamber, valve, and great vessel orientation. Wall thickness was measured using the Image-Pro 6.2 program (Media Cybernetics) calibrated to a reference marker captured at the same magnification. Embryo sections that included ventricular cavities, the LV free wall, the interventricular septum, and the RV free wall were selected for wall thickness measurement. The mean wall thickness from 4 serial sections was determined for each embryo.

Immunostaining. The sections were deparaffinized in xylenes, hydrated through alcohol series, and washed with water. Antigen retrieval was performed by boiling the samples in sodium citrate solution 3 times for 5 minutes. The endogenous peroxidase activity was quenched by incubating the 
sections in $3 \% \mathrm{H}_{2} \mathrm{O}_{2}$ for 10 minutes. Next, the sections were blocked with a blocking solution containing $2 \%$ BSA, $0.2 \%$ horse serum in PBS supplemented with $0.2 \% \mathrm{NP}-40$. The primary antibody was added in blocking solution, and the samples were incubated overnight. For immunofluorescence, the sections were incubated with fluorescein-conjugated secondary antibody (Invitrogen) together with DAPI for 1 hour at room temperature and were then washed. Coverslips were secured with Immu-Mount (Thermo Electron). For immunocytochemistry, the sections were incubated with biotin-conjugated secondary antibody $(\mathrm{BD})$ for 30 minutes at room temperature, washed, and incubated for 30 minutes with $\mathrm{ABC}$ reagent (Vector Laboratories). The sections were then washed and incubated in DAB solution (Zymed, Invitrogen) for 3 minutes and counterstained with hematoxylin (Zymed, Invitrogen). Finally, the slides were dehydrated, cleared in xylenes, and mounted with Permount (Biomeda). Antibody against phospho-histone $\mathrm{H} 3$ (Ser10) was from Upstate, $\beta$-catenin antibody was from $\mathrm{BD}$, and the other antibodies were from Santa Cruz Biotechnology Inc.

SPOT Imaging software (Diagnostic Instruments Inc.) was used to record immunohistochemistry images, and OpenLab or NIS Elements software was used to record immunofluorescence images.

Cardiomyocyte proliferation assay. Primary cultures of neonatal rat ventricular myocytes (NRVMs) were prepared from 2- to 4-day-old Sprague-Dawley rats as previously described (45). The cells were plated onto laminin-coated glass coverslips for immunofluorescence studies or onto Primaria cell culture dishes $(\mathrm{BD})$ for immunoblotting. Following desired treatments, the cells were either fixed with $4 \%$ paraformaldehyde, blocked with blocking solution, and stained for phospho-histone H3 (Ser10) as described above or lysed for immunoblotting as described previously (45).

Statistics. Rates of EB contraction were compared by $2 \times 2$ contingency table analysis using Fisher's exact test administered by the Prism statis- tical package (GraphPad Software). Data analysis was performed with 2 -tailed Student's $t$ test for unpaired samples and $\chi^{2}$ test for differences between observed and expected distributions. The significance of wall thickness difference and EB gene expression was determined by 1-way ANOVA, with multiple comparisons performed using the Holm-Sidak method. Statistical analysis of the ability to identify the genotype of heart sections correctly was done by a binomial test, in which a hypothesis test was performed against the null hypothesis that the success rate would be 0.5 (i.e., chance).

\section{Acknowledgments}

We are indebted to Flore Celeste for preparing and sectioning the embryos for this study, and we thank Abhijit Dasgupta for assistance with statistical analyses. We thank Richard Patten and Elena Kudravtseva for assistance in the quantitative real-time RT-PCR experiment. Grants from the American Heart Association, Canadian Institutes of Health Research, Finnish Heart Foundation, Paavo Nurmi Foundation, and NIH supported this work.

Received for publication May 19, 2008, and accepted in revised form August 18, 2008.

Address correspondence to: Thomas Force, Center for Translational Medicine, Jefferson Medical College, 1025 Walnut Street, Room 316, Philadelphia, Pennsylvania 19107, USA. Phone: (215) 503-9520; Fax: (215) 503-5731; E-mail: thomas.force@jefferson.edu.

Risto Kerkela and Lisa Kockeritz contributed equally to this work.
1. Gassmann, M., et al. 1995. Aberrant neural and cardiac development in mice lacking the ErbB4 neuregulin receptor. Nature. 378:390-394.

2. Lee, K.F., et al. 1995. Requirement for neuregulin receptor erbB2 in neural and cardiac development. Nature. 378:394-398.

3. Meyer, D., and Birchmeier, C. 1995. Multiple essential functions of neuregulin in development. Nature. 378:386-390.

4. Hertig, C.M., Kubalak, S.W., Wang, Y., and Chien, K.R. 1999. Synergistic roles of neuregulin-1 and insulin-like growth factor-I in activation of the phosphatidylinositol 3-kinase pathway and cardiac chamber morphogenesis. J. Biol. Chem. 274:37362-37369.

5. Lavine, K.J., et al. 2005. Endocardial and epicardial derived FGF signals regulate myocardial proliferation and differentiation in vivo. Dev. Cell. 8:85-95.

6. Teichmann, U., and Kessel, M. 2004. Highly restricted BMP10 expression in the trabeculating myocardium of the chick embryo. Dev. Genes Evol. 214:96-98.

7. Cai, C.L., et al. 2005. T-box genes coordinate regional rates of proliferation and regional specification during cardiogenesis. Development. 132:2475-2487.

8. Bushdid, P.B., Osinska, H., Waclaw, R.R., Molkentin, J.D., and Yutzey, K.E. 2003. NFATc3 and NFATc4 are required for cardiac development and mitochondrial function. Circ. Res. 92:1305-1313.

9. Hurlin, P.J. 2005. N-Myc functions in transcription and development. Birth Defects Res. C Embryo Today. 75:340-352.

10. Moens, C.B., Stanton, B.R., Parada, L.F., and Rossant, J. 1993. Defects in heart and lung development in compound heterozygotes for two different targeted mutations at the N-myc locus. Development. 119:485-499.

11. Zeisberg, E.M., et al. 2005. Morphogenesis of the right ventricle requires myocardial expression of Gata4. J. Clin. Invest. 115:1522-1531.

12. Sundgren, N.C., et al. 2003. Extracellular signalregulated kinase and phosphoinositol-3 kinase mediate IGF-1 induced proliferation of fetal sheep cardiomyocytes. Am. J. Physiol. Regul. Integr. Comp. Physiol. 285:R1481-R1489.

13. McDevitt, T.C., Laflamme, M.A., and Murry, C.E. 2005. Proliferation of cardiomyocytes derived from human embryonic stem cells is mediated via the IGF/PI 3-kinase/Akt signaling pathway. J. Mol. Cell. Cardiol. 39:865-873.

14. Woodgett, J.R. 1990. Molecular cloning and expression of glycogen synthase kinase-3/factor A. EMBOJ. 9:2431-2438

15. Frame, S., and Cohen, P. 2001. GSK3 takes centre stage more than 20 years after its discovery. Biochem. J. 359:1-16.

16. Hurlstone, A.F., et al. 2003. The Wnt/beta-catenin pathway regulates cardiac valve formation. Nature. 425:633-637.

17. Lickert, H., et al. 2002. Formation of multiple hearts in mice following deletion of beta-catenin in the embryonic endoderm. Dev. Cell. 3:171-181.

18. Liebner, S., et al. 2004. Beta-catenin is required for endothelial-mesenchymal transformation during heart cushion development in the mouse. J. Cell Biol. 166:359-367.

19. Eisenberg, L.M., and Eisenberg, C.A. 2006. Wnt signal transduction and the formation of the myocardium. Dev. Biol. 293:305-315.

20. Hamblet, N.S., et al. 2002. Dishevelled 2 is essential for cardiac outflow tract development, somite segmentation and neural tube closure. Development. 129:5827-5838.

21. Kioussi, C., et al. 2002. Identification of a Wnt/Dvl/ beta-Catenin --> Pitx2 pathway mediating cell-typespecific proliferation during development. Cell. 111:673-685.
22. Doble, B.W., Patel, S., Wood, G.A., Kockeritz, L.K., and Woodgett, J.R. 2007. Functional redundancy of GSK-3alpha and GSK-3beta in Wnt/beta-catenin signaling shown by using an allelic series of embryonic stem cell lines. Dev. Cell. 12:957-971.

23. Diehl, J.A., Cheng, M., Roussel, M.F., and Sherr, C.J. 1998. Glycogen synthase kinase-3beta regulates cyclin D1 proteolysis and subcellular localization. Genes Dev. 12:3499-3511.

24. Sears, R., et al. 2000. Multiple Ras-dependent phosphorylation pathways regulate Myc protein stability. Genes Dev. 14:2501-2514.

25. Yang, K., et al. 2006. Glycogen synthase kinase 3 has a limited role in cell cycle regulation of cyclin D1 levels. BMC Cell Biol. 7:33.

26. Guo, Y., et al. 2005. Phosphorylation of cyclin D1 at Thr 286 during S phase leads to its proteasomal degradation and allows efficient DNA synthesis. Oncogene. 24:2599-2612.

27. Okabe, H., et al. 2006. A critical role for FBXW8 and MAPK in cyclin D1 degradation and cancer cell proliferation. PLOS ONE. 1:e128.

28. Kessler, R.C., et al. 2005. Lifetime prevalence and age-of-onset distributions of DSM-IV disorders in the National Comorbidity Survey Replication. Arch. Gen. Psychiatry. 62:593-602.

29. Rowe, M.K., Wiest, C., and Chuang, D.M. 2007. GSK-3 is a viable potential target for therapeutic intervention in bipolar disorder. Neurosci. Biobehav. Rev. 31:920-931.

30. Cohen, L.S., Friedman, J.M., Jefferson, J.W., Johnson, E.M., and Weiner, M.L. 1994. A reevaluation of risk of in utero exposure to lithium. JAMA. 271:146-150.

31. Hoeflich, K.P., et al. 2000. Requirement for glycogen synthase kinase-3beta in cell survival and NFkappaB activation. Nature. 406:86-90.

32. Liu, K.J., Arron, J.R., Stankunas, K., Crabtree, G.R., and Longaker, M.T. 2007. Chemical rescue of cleft 
palate and midline defects in conditional GSK3beta mice. Nature. 446:79-82.

33. Pfeffer, K., et al. 1993. Mice deficient for the 55 $\mathrm{kd}$ tumor necrosis factor receptor are resistant to endotoxic shock, yet succumb to L. monocytogenes infection. Cell. 73:457-467.

34. Michael, A., et al. 2004. Glycogen synthase kinase3 beta regulates growth, calcium homeostasis, and diastolic function in the heart. J. Biol. Chem. 279:21383-21393.

35. Antos, C.L., et al. 2002. Activated glycogen synthase- 3 beta suppresses cardiac hypertrophy in vivo. Proc. Natl. Acad. Sci. U. S. A. 99:907-912.

36. Sanbe, A., et al. 2003. Reengineering inducible cardiac-specific transgenesis with an attenuated myosin heavy chain promoter. Circ. Res. 92:609-616.

37. Hirotani, S., et al. 2007. Inhibition of glycogen synthase kinase 3 beta during heart failure is protective. Circ. Res. 101:1164-1174.

38. Rivera-Feliciano, J., et al. 2006. Development of heart valves requires Gata4 expression in endothelialderived cells. Development. 133:3607-3618.

39. Welcker, M., et al. 2003. Multisite phosphorylation by Cdk2 and GSK3 controls cyclin E degradation. Mol. Cell. 12:381-392.

40. Beals, C.R., Sheridan, C.M., Turck, C.W., Gardner, P., and Crabtree, G.R. 1997. Nuclear export of NF-ATc enhanced by glycogen synthase kinase- 3 . Science. 275:1930-1934.

41. Morisco, C., et al. 2001. Glycogen synthase kinase 3 beta regulates GATA4 in cardiac myocytes. J. Biol. Chem. 276:28586-28597.

42. Ueno, S., et al. 2007. Biphasic role for Wnt/betacatenin signaling in cardiac specification in zebrafish and embryonic stem cells. Proc. Natl. Acad. Sci.U.S. A. 104:9685-9690.
43. Lee, H.C., et al. 2007. Glycogen synthase kinase 3 alpha and 3 beta have distinct functions during cardiogenesis of zebrafish embryo. BMC Dev. Biol. 7:93.

44. Tsai, J.N., Lee, C.H., Jeng, H., Chi, W.K., and Chang, W.C. 2000. Differential expression of glycogen synthase kinase 3 genes during zebrafish embryogenesis. Mech. Dev. 91:387-391.

45. Haq, S., et al. 2000. Glycogen synthase kinase-3beta is a negative regulator of cardiomyocyte hypertrophy. J. Cell Biol. 151:117-130.

46. Luckey, S.W., et al. 2007. Blocking cardiac growth in hypertrophic cardiomyopathy induces cardiac dysfunction and decreased survival only in males. Am. J. Physiol. Heart Circ. Physiol. 292:H838-H845.

47. Ahuja, P., Sdek, P., and MacLellan, W.R. 2007. Cardiac myocyte cell cycle control in development, disease, and regeneration. Physiol. Rev. 87:521-544.

48. Kozar, K., and Sicinski, P. 2005. Cell cycle progression without cyclin D-CDK4 and cyclin D-CDK6 complexes. Cell Cycle. 4:388-391.

49. Berthet, C., et al. 2006. Combined loss of Cdk2 and $\mathrm{Cdk} 4$ results in embryonic lethality and $\mathrm{Rb}$ hypophosphorylation. Dev. Cell. 10:563-573.

50. Xin, M., et al. 2006. A threshold of GATA4 and GATA6 expression is required for cardiovascular development. Proc. Natl. Acad. Sci. U. S. A. 103:11189-11194.

51. Aghdam, S.Y., and Barger, S.W. 2007. Glycogen synthase kinase-3 in neurodegeneration and neuroprotection: lessons from lithium. Curr. Alzheimer Res. 4:21-31.

52. Dugo, L., Collin, M., and Thiemermann, C. 2007. Glycogen synthase kinase 3 beta as a target for the therapy of shock and inflammation. Shock. 27:113-123.

53. MacAulay, K., et al. 2007. Glycogen synthase kinase 3alpha-specific regulation of murine hepatic glycogen metabolism. Cell Metab. 6:329-337.

54. Medina, M., and Castro, A. 2008. Glycogen synthase kinase-3 (GSK-3) inhibitors reach the clinic. Curr. Opin. Drug Discov. Devel. 11:533-543.

55. Reis, M., and Kallen, B. 2008. Maternal use of antipsychotics in early pregnancy and delivery outcome. J. Clin. Psychopharmacol. 28:279-288.

56. Chen, J., et al. 2008. Molecular effects of lithium exposure during mouse and chick gastrulation and subsequent valve dysmorphogenesis. Birth Defects Res. A Clin. Mol. Teratol. 82:508-518.

57. O'Brien, W.T., et al. 2004. Glycogen synthase kinase-3beta haploinsufficiency mimics the behavioral and molecular effects of lithium. J. Neurosci. 24:6791-6798.

58. Berridge, M.J. 1989. The Albert Lasker Medical Awards. Inositol trisphosphate, calcium, lithium, and cell signaling. JAMA. 262:1834-1841.

59. Bersudsky, Y., et al. 2008. Glycogen synthase kinase3 beta heterozygote knockout mice as a model of findings in postmortem schizophrenia brain or as a model of behaviors mimicking lithium action: negative results. Behav. Pharmacol. 19:217-224.

60. Beaulieu, J.M., et al. 2008. A beta-arrestin 2 signaling complex mediates lithium action on behavior. Cell. 132:125-136.

61. Pfaffl, M.W. 2001. A new mathematical model for relative quantification in real-time RT-PCR. Nucleic Acids Res. 29:e45.

62. Nagy, A. 2000. Cre recombinase: the universal reagent for genome tailoring. Genesis. 26:99-109.

63. Chen, X., et al. 2006. The beta-catenin/T-cell factor/lymphocyte enhancer factor signaling pathway is required for normal and stress-induced cardiac hypertrophy. Mol. Cell. Biol. 26:4462-4473. 